\title{
Quantitative-Voronovskaya and
}

\section{Grüss-Voronovskaya type theorems for Szász-Durrmeyer type operators blended with multiple Appell polynomials}

Trapti Neer* and Purshottam Narain Agrawal

${ }^{*}$ Correspondence:

triptineeriitr@gmail.com

Department of Mathematics, Indian

Institute of Technology Roorkee,

Roorkee, 247667, India

\section{Springer}

\begin{abstract}
In this paper, we establish a link between the Szász-Durrmeyer type operators and multiple Appell polynomials. We study a quantitative-Voronovskaya type theorem in terms of weighted modulus of smoothness using sixth order central moment and Grüss-Voronovskaya type theorem. We also establish a local approximation theorem by means of the Steklov means in terms of the first and the second order modulus of continuity and Voronovskaya type asymtotic theorem. Further, we discuss the degree of approximation by means of the weighted spaces. Lastly, we find the rate of approximation of functions having a derivative of bounded variation.
\end{abstract}

MSC: Primary 41A25; 41A36

Keywords: Steklov mean; first and second order modulus of continuity; weighted modulus of continuity; Grüss-Voronovskaya type theorem; functions of bounded variation

\section{Introduction}

For $f \in C\left(R_{0}^{+}\right)$and $x \in R_{0}^{+}\left(R_{0}^{+}=[0, \infty)\right)$, Szász [1] introduced the well-known operators

$$
S_{n}(f ; x)=e^{-n x} \sum_{k=0}^{\infty} \frac{(n x)^{k}}{k !} f\left(\frac{k}{n}\right)
$$

such that $S_{n}(|f| ; x)<\infty$. Several generalizations of Szász operators have been introduced in the literature and authors have studied their approximation properties. In [2], the author considered Baskakov-Szász type operators and studied the rate of convergence for absolutely continuous functions having a derivative equivalent with a function of bounded variation. In [3], the authors introduced the $q$-Baskakov-Durrmeyer type operators and studied the rate of convergence and the weighted approximation properties. In [4] the authors proposed the $\beta$-operators based on $q$-integers and established some direct theorems by means of modulus of continuity and also studied the weighted approximation and better approximation using King type approach. For exhaustive literature on approximation by linear positive operators one can refer to [5-7] and the references therein.

(c) The Author(s) 2017. This article is distributed under the terms of the Creative Commons Attribution 4.0 International License (http://creativecommons.org/licenses/by/4.0/), which permits unrestricted use, distribution, and reproduction in any medium, provided you give appropriate credit to the original author(s) and the source, provide a link to the Creative Commons license, and indicate if changes were made. 
Now let us recall some results on multiple Appell polynomials [8]. Let $g(z)=\sum_{n=0}^{\infty} a_{n} z^{n}$, $g(1) \neq 1$, be an analytic function in the disc $|z| \leq r, r>1$ and $p_{k}(x)$ be the Appell polynomials having the generating function $g(u) e^{u x}=\sum_{k=0}^{\infty} p_{k}(x) u^{k}$, with $g(1) \neq 0$ and $p_{k}(x) \geq 0$, $\forall x \in R_{0}^{+}$.

Jakimovski and Leviatan [9] proposed a generalization of Szász-Mirakjan operators by means of the Appell polynomials as follows:

$$
P_{n}(f ; x)=\frac{e^{-n x}}{g(1)} \sum_{k=0}^{\infty} p_{k}(n x) f\left(\frac{k}{n}\right)
$$

For $g(u)=1$, these operators reduce to Szász-Mirakjan operators (1).

A set of polynomials $\left\{p_{k_{1}, k_{2}}(x)\right\}_{k_{1}, k_{2}=0}^{\infty}$ with degree $k_{1}+k_{2}$ for $k_{1}, k_{2} \geq 0$ is called multiple polynomial system (multiple PS) and a multiple PS is called multiple Appell if it is generated by the relation

$$
A\left(t_{1}, t_{2}\right) e^{x\left(t_{1}+t_{2}\right)}=\sum_{k_{1}=0}^{\infty} \sum_{k_{2}=0}^{\infty} \frac{p_{k_{1}, k_{2}}(x)}{k_{1} ! k_{2} !} t_{1}^{k_{1}} t_{2}^{k_{2}},
$$

where $A$ is given by

$$
A\left(t_{1}, t_{2}\right)=\sum_{k_{1}=0}^{\infty} \sum_{k_{2}=0}^{\infty} \frac{a_{k_{1}, k_{2}}}{k_{1} ! k_{2} !} t_{1}^{k_{1}} t_{2}^{k_{2}}
$$

with $A(0,0)=a_{0,0} \neq 0$.

Theorem 1.1 For multiple PS, $\left\{p_{k_{1}, k_{2}}(x)\right\}_{k_{1}, k_{2}=0}^{\infty}$, the following statements are equivalent:

(a) $\left\{p_{k_{1}, k_{2}}(x)\right\}_{k_{1}, k_{2}=0}^{\infty}$ is a set of multiple Appell polynomials.

(b) There exists a sequence $\left\{a_{k_{1}, k_{2}}\right\}_{k_{1}, k_{2}=0}^{\infty}$ with $a_{0,0} \neq 0$ such that

$$
p_{k_{1}, k_{2}}(x)=\sum_{r_{1}=0}^{\infty} \sum_{r_{2}=0}^{\infty}\left(\begin{array}{l}
k_{1} \\
r_{1}
\end{array}\right)\left(\begin{array}{l}
k_{2} \\
r_{2}
\end{array}\right) a_{k_{1}-r_{1}, k_{2}-r_{2}} x^{r_{1}+r_{2}}
$$

(c) For every $k_{1}+k_{2} \geq 1$, we have

$$
p_{k_{1}, k_{2}}^{\prime}(x)=k_{1} p_{k_{1}-1, k_{2}}(x)+k_{2} p_{k_{1}, k_{2}-1}(x)
$$

Varma [10] defined a sequence of linear positive operators for any $f \in C\left(R_{0}^{+}\right)$, by

$$
K_{n}(f ; x)=\frac{e^{-n x}}{A(1,1)} \sum_{k_{1}=0}^{\infty} \sum_{k_{2}=0}^{\infty} \frac{p_{k_{1}, k_{2}}\left(\frac{n x}{2}\right)}{k_{1} ! k_{2} !} f\left(\frac{k_{1}+k_{2}}{n}\right),
$$

provided $A(1,1) \neq 0, \frac{a_{k_{1}, k_{2}}}{A(1,1)} \geq 0$ for $k_{1}, k_{2} \in \mathbb{N}$, and the series (3) and(4) converge for $\left|t_{1}\right|<R_{1}$, $\left|t_{2}\right|<R_{2}\left(R_{1}, R_{2}>1\right)$, respectively.

For $\alpha>0, \rho>0$ and $f: R_{0}^{+} \rightarrow \mathbb{R}$, being integrable function, Pǎltǎnea [11] defined a modification of the Szász operators by

$$
L_{\alpha}^{\rho}(f ; x)=\sum_{k=1}^{\infty} s_{\alpha, k}(x) \int_{0}^{\infty} \frac{\alpha \rho e^{-\alpha \rho t}(\alpha \rho t)^{(k) \rho-1}}{\Gamma(k) \rho} f(t) d t+e^{-\alpha x} f(0), \quad x \in R_{0}^{+} .
$$


Motivated by [11], for $f \in C_{E}\left(R_{0}^{+}\right)$, the space of all continuous functions satisfying $|f(t)| \leq$ $K e^{a t}(t \geq 0)$ for some positive constant $K$ and $a$, we propose an approximation method by linking the operators (6) and the multiple Appell polynomials by

$$
\begin{aligned}
\mathcal{L}_{n}^{\rho}(f ; x)= & \frac{e^{-n x}}{A(1,1)} \sum_{\substack{k_{1} \\
k_{1}+k_{2} \geq 1}} \sum_{k_{2}} \frac{p_{k_{1}, k_{2}}\left(\frac{n x}{2}\right)}{k_{1} ! k_{2} !} \int_{0}^{\infty} \frac{n \rho e^{-n \rho t}(n \rho t)^{\left(k_{1}+k_{2}\right) \rho-1}}{\Gamma\left(k_{1}+k_{2}\right) \rho} f(t) d t \\
& +\frac{e^{-n x}}{A(1,1)} p_{0,0}\left(\frac{n x}{2}\right) f(0),
\end{aligned}
$$

and establish a quantitative Voronovskaya type theorem, a Grüss Voronovskaya type theorem, a local approximation theorem by means of the Steklov mean, a Voronovskaya type asymptotic theorem and error estimates for several weighted spaces. Lastly, we study the rate of convergence of functions having a derivative of bounded variation.

\section{Basic results}

In order to prove the main results of the paper, we shall need the following auxiliary results.

Lemma 2.1 For $K_{n}\left(t^{i} ; x\right), i=0,1,2,3,4$, we have

(i) $K_{n}(1 ; x)=1$,

(ii) $K_{n}(t ; x)=x+\frac{A_{t_{1}}(1,1)+A_{t_{2}}(1,1)}{n A(1,1)}$,

(iii) $K_{n}\left(t^{2} ; x\right)=x^{2}+\frac{x}{n}\left\{1+\frac{2}{A(1,1)}\left(A_{t_{1}}(1,1)+A_{t_{2}}(1,1)\right)\right\}$

$$
+\frac{1}{n^{2} A(1,1)}\left\{A_{t_{1}}(1,1)+A_{t_{2}}(1,1)+A_{t_{1} t_{1}}(1,1)+2 A_{t_{1} t_{2}}(1,1)+A_{t_{2} t_{2}}(1,1)\right\}
$$

(iv) $K_{n}\left(t^{3} ; x\right)=x^{3}+\frac{3 x^{2}}{n}\left\{1+\frac{1}{A(1,1)}\left(A_{t_{1}}(1,1)+A_{t_{2}}(1,1)\right)\right\}+\frac{x}{n^{2}}\{1$

$$
\begin{aligned}
& \left.+\frac{3}{A(1,1)}\left(2 A_{t_{1}}(1,1)+2 A_{t_{2}}(1,1)+A_{t_{1} t_{1}}(1,1)+2 A_{t_{1} t_{2}}(1,1)+A_{t_{2} t_{2}}(1,1)\right)\right\} \\
& +\frac{1}{n^{3} A(1,1)}\left\{A_{t_{1}}(1,1)+A_{t_{2}}(1,1)+3 A_{t_{1} t_{1}}(1,1)+6 A_{t_{1} t_{2}}(1,1)\right. \\
& \left.+3 A_{t_{2} t_{2}}(1,1)+A_{t_{1} t_{1} t_{1}}(1,1)+A_{t_{2} t_{2} t_{2}}(1,1)+3 A_{t_{1} t_{1} t_{2}}(1,1)+3 A_{t_{2} t_{2} t_{1}}(1,1)\right\},
\end{aligned}
$$

(v) $K_{n}\left(t^{4} ; x\right)=x^{4}+\frac{x^{3}}{n}\left\{6+\frac{4}{A(1,1)}\left(A_{t_{1}}(1,1)+A_{t_{2}}(1,1)\right)\right\}+\frac{x^{2}}{n^{2}}\{7$

$$
\begin{aligned}
& \left.+\frac{6}{A(1,1)}\left(3 A_{t_{1}}(1,1)+3 A_{t_{2}}(1,1)+A_{t_{1} t_{1}}(1,1)+2 A_{t_{1} t_{2}}(1,1)+A_{t_{2} t_{2}}(1,1)\right)\right\} \\
& +\frac{x}{n^{3}}\left\{1+\frac{1}{A(1,1)}\left(14 A_{t_{1}}(1,1)+14 A_{t_{2}}(1,1)+18 A_{t_{1} t_{1}}(1,1)+36 A_{t_{1} t_{2}}(1,1)\right.\right. \\
& +18 A_{t_{2} t_{2}}(1,1)+4 A_{t_{1} t_{1} t_{1}}(1,1)+4 A_{t_{2} t_{2} t_{2}}(1,1)+12 A_{t_{1} t_{1} t_{2}}(1,1) \\
& \left.\left.+12 A_{t_{2} t_{2} t_{1}}(1,1)\right)\right\}+\frac{1}{n^{4} A(1,1)}\left\{A_{t_{1}}(1,1)+A_{t_{2}}(1,1)+7 A_{t_{1} t_{1}}(1,1)\right. \\
& +14 A_{t_{1} t_{2}}(1,1)+7 A_{t_{2} t_{2}}(1,1)+6 A_{t_{1} t_{1} t_{1}}(1,1)+6 A_{t_{2} t_{2} t_{2}}(1,1)
\end{aligned}
$$




$$
\begin{aligned}
& +18 A_{t_{1} t_{1} t_{2}}(1,1)+18 A_{t_{2} t_{2} t_{1}}(1,1)+A_{t_{1} t_{1} t_{1} t_{1}}(1,1)+A_{t_{2} t_{2} t_{2} t_{2}}(1,1) \\
& \left.+4 A_{t_{1} t_{1} t_{1} t_{2}}(1,1)+4 A_{t_{2} t_{2} t_{2} t_{1}}(1,1)+6 A_{t_{1} t_{1} t_{2} t_{2}}(1,1)\right\}
\end{aligned}
$$

The values of the moments $K_{n}\left(t^{i} ; x\right)$ for $i=0,1,2$ are given in [10] while the values of $K_{n}\left(t^{i} ; x\right)$ for $i=3,4$ have been obtained by us after simple calculations and hence the details are omitted.

Lemma 2.2 For the sequence of linear positive operators $\mathcal{L}_{n}^{\rho}\left(t^{i} ; x\right), i=0,1,2,3,4$, we find

(i) $\quad \mathcal{L}_{n}^{\rho}(1 ; x)=1$,

(ii) $\mathcal{L}_{n}^{\rho}(t ; x)=x+\frac{A_{t_{1}}(1,1)+A_{t_{2}}(1,1)}{n A(1,1)}$,

(iii) $\quad \mathcal{L}_{n}^{\rho}\left(t^{2} ; x\right)=x^{2}+\frac{x}{n}\left\{\left(1+\frac{1}{\rho}\right)+\frac{2}{A(1,1)}\left(A_{t_{1}}(1,1)+A_{t_{2}}(1,1)\right)\right\}$

$$
\begin{aligned}
& +\frac{1}{n^{2} A(1,1)}\left\{\left(1+\frac{1}{\rho}\right)\left(A_{t_{1}}(1,1)+A_{t_{2}}(1,1)\right)+A_{t_{1} t_{1}}(1,1)\right. \\
& \left.+2 A_{t_{1} t_{2}}(1,1)+A_{t_{2} t_{2}}(1,1)\right\},
\end{aligned}
$$

(iv) $\mathcal{L}_{n}^{\rho}\left(t^{3} ; x\right)=x^{3}+\frac{3 x^{2}}{n}\left\{\left(1+\frac{1}{\rho}\right)+\frac{1}{A(1,1)}\left(A_{t_{1}}(1,1)+A_{t_{2}}(1,1)\right)\right\}$

$$
\begin{aligned}
& +\frac{x}{n^{2}}\left\{\left(1+\frac{3}{\rho}+\frac{2}{\rho^{2}}\right)+\frac{3}{A(1,1)}\left(2\left(1+\frac{1}{\rho}\right)\left(A_{t_{1}}(1,1)+A_{t_{2}}(1,1)\right)\right.\right. \\
& \left.\left.+A_{t_{1} t_{1}}(1,1)+2 A_{t_{1} t_{2}}(1,1)+A_{t_{2} t_{2}}(1,1)\right)\right\} \\
& +\frac{1}{n^{3} A(1,1)}\left\{\left(1+\frac{3}{\rho}+\frac{2}{\rho^{2}}\right)\left(A_{t_{1}}(1,1)+A_{t_{2}}(1,1)\right)\right. \\
& +3\left(1+\frac{1}{\rho}\right)\left(A_{t_{1} t_{1}}(1,1)+2 A_{t_{1} t_{2}}(1,1)+A_{t_{2} t_{2}}(1,1)\right)+A_{t_{1} t_{1} t_{1}}(1,1) \\
& \left.+A_{t_{2} t_{2} t_{2}}(1,1)+3 A_{t_{1} t_{1} t_{2}}(1,1)+3 A_{t_{2} t_{2} t_{1}}(1,1)\right\}
\end{aligned}
$$

(v) $\mathcal{L}_{n}^{\rho}\left(t^{4} ; x\right)=x^{4}+\frac{x^{3}}{n}\left\{6\left(1+\frac{1}{\rho}\right)+\frac{4}{A(1,1)}\left(A_{t_{1}}(1,1)+A_{t_{2}}(1,1)\right)\right\}$

$$
\begin{aligned}
& +\frac{x^{2}}{n^{2}}\left\{\left(7+\frac{18}{\rho}+\frac{11}{\rho^{2}}\right)+\frac{6}{A(1,1)}\left(3\left(1+\frac{1}{\rho}\right)\left(A_{t_{1}}(1,1)+A_{t_{2}}(1,1)\right)\right.\right. \\
& \left.\left.+A_{t_{1} t_{1}}(1,1)+2 A_{t_{1} t_{2}}(1,1)+A_{t_{2} t_{2}}(1,1)\right)\right\}+\frac{x}{n^{3}}\left\{\left(1+\frac{6}{\rho}+\frac{11}{\rho^{2}}+\frac{6}{\rho^{3}}\right)\right. \\
& +\frac{1}{A(1,1)}\left(\left(14+\frac{36}{\rho}+\frac{22}{\rho^{2}}\right)\left(A_{t_{1}}(1,1)+A_{t_{2}}(1,1)\right)\right. \\
& +18\left(1+\frac{1}{\rho}\right)\left(A_{t_{1} t_{1}}(1,1)+2 A_{t_{1} t_{2}}(1,1)+A_{t_{2} t_{2}}(1,1)\right)+4 A_{t_{1} t_{1} t_{1}}(1,1) \\
& \left.\left.+4 A_{t_{2} t_{2} t_{2}}(1,1)+12 A_{t_{1} t_{1} t_{2}}(1,1)+12 A_{t_{2} t_{2} t_{1}}(1,1)\right)\right\}
\end{aligned}
$$




$$
\begin{aligned}
& +\frac{1}{n^{4} A(1,1)}\left\{\left(1+\frac{6}{\rho}+\frac{11}{\rho^{2}}+\frac{6}{\rho^{3}}\right)\left(A_{t_{1}}(1,1)+A_{t_{2}}(1,1)\right)\right. \\
& +\left(7+\frac{18}{\rho}+\frac{11}{\rho^{2}}\right)\left(A_{t_{1} t_{1}}(1,1)+2 A_{t_{1} t_{2}}(1,1)+A_{t_{2} t_{2}}(1,1)\right) \\
& +6\left(1+\frac{1}{\rho}\right)\left(A_{t_{1} t_{1} t_{1}}(1,1)+A_{t_{2} t_{2} t_{2}}(1,1)+3 A_{t_{1} t_{1} t_{2}}(1,1)+3 A_{t_{2} t_{2} t_{1}}(1,1)\right) \\
& +A_{t_{1} t_{1} t_{1} t_{1}}(1,1)+A_{t_{2} t_{2} t_{2} t_{2}}(1,1)+4 A_{t_{1} t_{1} t_{1} t_{2}}(1,1) \\
& \left.+4 A_{t_{2} t_{2} t_{2} t_{1}}(1,1)+6 A_{t_{1} t_{1} t_{2} t_{2}}(1,1)\right\} .
\end{aligned}
$$

Consequently,

$$
\begin{aligned}
\mathcal{L}_{n}^{\rho}\left((t-x)^{2} ; x\right)= & \frac{x}{n}\left(1+\frac{1}{\rho}\right)+\frac{1}{n^{2} A(1,1)}\left\{\left(1+\frac{1}{\rho}\right)\left(A_{t_{1}}(1,1)+A_{t_{2}}(1,1)\right)\right. \\
& \left.+A_{t_{1} t_{1}}(1,1)+2 A_{t_{1} t_{2}}(1,1)+A_{t_{2} t_{2}}(1,1)\right\} \\
\leq & \frac{C}{n}\left(1+\frac{1}{\rho}\right)(1+x) \\
= & \delta_{n, \rho}^{2}(x) \quad(\text { say }),
\end{aligned}
$$

where

$$
C=\max \left(1, \frac{\left|A_{t_{1}}(1,1)\right|+\left|A_{t_{2}}(1,1)\right|+\left|A_{t_{1}, t_{1}}(1,1)\right|+2\left|A_{t_{1}, t_{2}}(1,1)\right|+\left|A_{t_{2}, t_{2}}(1,1)\right|}{|A(1,1)|}\right)
$$

and

$$
\begin{aligned}
\mathcal{L}_{n}^{\rho}\left((t-x)^{4} ; x\right)= & \frac{3 x^{2}}{n^{2}}\left\{1+\frac{2}{\rho}+\frac{1}{\rho^{2}}\right\}+\frac{x}{n^{3}}\left[\left(1+\frac{6}{\rho}+\frac{11}{\rho^{2}}+\frac{6}{\rho^{3}}\right)\right. \\
& +\frac{1}{A(1,1)}\left\{\left(13+\frac{33}{\rho}+\frac{20}{\rho^{2}}\right)\left(A_{t_{1}}(1,1)+A_{t_{2}}(1,1)\right)\right. \\
& +6\left(1+\frac{1}{\rho}\right)\left(A_{t_{1} t_{1}}(1,1)+2 A_{t_{1} t_{2}}(1,1)+A_{t_{2} t_{2}}(1,1)\right) \\
& \left.\left.-6 A_{t_{1} t_{1} t_{1}}(1,1)-6 A_{t_{2} t_{2} t_{2}}(1,1)\right\}\right] .
\end{aligned}
$$

The expression for $\mathcal{L}_{n}^{\rho}\left((t-x)^{6} ; x\right)$ has not been included in Lemma 2.2 because it is very lengthy and complicated. It will be required to prove the quantitative Voronovskaya type theorem.

Remark 2.3 From Lemma 2.2, we obtain

$$
\begin{aligned}
& \lim _{n \rightarrow \infty} n \mathcal{L}_{n}^{\rho}((t-x) ; x)=\frac{A_{t_{1}}(1,1)+A_{t_{2}}(1,1)}{A(1,1)}, \\
& \lim _{n \rightarrow \infty} n \mathcal{L}_{n}^{\rho}\left((t-x)^{2} ; x\right)=x\left(1+\frac{1}{\rho}\right),
\end{aligned}
$$




$$
\begin{aligned}
& \lim _{n \rightarrow \infty} n^{2} \mathcal{L}_{n}^{\rho}\left((t-x)^{4} ; x\right)=3 x^{2}\left(1+\frac{2}{\rho}+\frac{1}{\rho^{2}}\right) \\
& \lim _{n \rightarrow \infty} n^{3} \mathcal{L}_{n}^{\rho}\left((t-x)^{6} ; x\right)=15 x^{3}\left(1+\frac{3}{\rho}+\frac{3}{\rho^{2}}+\frac{1}{\rho^{3}}\right)
\end{aligned}
$$

\section{Main results}

Theorem 3.1 Let $f \in C_{E}\left(R_{0}^{+}\right)$. Then $\lim _{n \rightarrow \infty} \mathcal{L}_{n}^{\rho}(f ; x)=f(x)$ uniformly on each compact subset of $R_{0}^{+}$.

Proof Considering Lemma 2.2, it follows that $\lim _{n \rightarrow \infty} \mathcal{L}_{n}^{\rho}\left(t^{i} ; x\right)=x^{i}, i=0,1,2$, uniformly on every compact subset of $R_{0}^{+}$. Applying the Bohman Korovkin theorem, we obtain the desired result.

For $f \in C_{B}\left(R_{0}^{+}\right)$, the space of bounded and continuous functions on $R_{0}^{+}$endowed with the norm $\|f\|=\sup _{x \in R_{0}^{+}}|f(x)|$, the first and second order modulus of continuity are, respectively, defined as

$$
\begin{aligned}
& \omega(f ; \delta)=\sup _{0<|h| \leq \delta} \sup _{x, x+h \in R_{0}^{+}}|f(x+h)-f(x)|, \\
& \omega_{2}(f ; \delta)=\sup _{0<|h| \leq \delta} \sup _{x, x+h, x+2 h \in R_{0}^{+}}|f(x+2 h)-2 f(x+h)+f(x)|, \quad \delta>0 .
\end{aligned}
$$

Further, for $f \in C_{B}\left(R_{0}^{+}\right)$, the Steklov mean of second order [12] is defined as

$$
f_{h}(x)=\frac{4}{h^{2}} \int_{0}^{\frac{h}{2}} \int_{0}^{\frac{h}{2}}[2 f(x+u+v)-f(x+2(u+v))] d u d v, \quad h>0
$$

Hence

$$
\begin{aligned}
& f(x)-f_{h}(x)=\frac{4}{h^{2}} \int_{0}^{h / 2} \int_{0}^{h / 2} \triangle_{u+1}^{2} f(x) d u d \nu, \quad \text { and } \\
& f_{h}^{\prime \prime}(x)=\frac{1}{h^{2}}\left(8 \triangle_{h / 2}^{2} f(x)-\triangle_{h}^{2} f(x)\right) .
\end{aligned}
$$

Thus, it follows that

$$
\left\|f_{h}-f\right\| \leq \omega_{2}(f, h)
$$

Further, $f_{h}^{\prime}, f_{h}^{\prime \prime} \in C_{B}\left(R_{0}^{+}\right)$and

$$
\left\|f_{h}^{\prime}\right\| \leq \frac{5}{h} \omega(f, h), \quad\left\|f_{h}^{\prime \prime}\right\| \leq \frac{9}{h^{2}} \omega_{2}(f, h) .
$$

Theorem 3.2 For $f \in C_{B}\left(R_{0}^{+}\right)$and $x \in R_{0}^{+}$, we have

$$
\left|\mathcal{L}_{n}^{\rho}(f ; x)-f(x)\right| \leq 5 \omega\left(f ; \delta_{n, \rho}(x)\right)+\frac{13}{2} \omega_{2}\left(f ; \delta_{n, \rho}(x)\right)
$$

where $\delta_{n, \rho}(x)$ is defined by equation (7). 
Proof Using the Steklov mean $f_{h}$ defined by (12), we may write

$$
\begin{aligned}
\left|\mathcal{L}_{n}^{\rho}(f ; x)-f(x)\right| \leq & \left|\mathcal{L}_{n}^{\rho}\left(\left(f-f_{h}\right) ; x\right)\right|+\left|\mathcal{L}_{n}^{\rho}\left(f_{h}(t)-f_{h}(x) ; x\right)\right| \\
& +\left|f_{h}(x)-f(x)\right| .
\end{aligned}
$$

Applying Lemma 2.2, we have

$$
\left\|\mathcal{L}_{n}^{\rho}(f)\right\| \leq\|f\|
$$

Using inequality (16) and equation (13), we have

$$
\begin{aligned}
\left|\mathcal{L}_{n}^{\rho}\left(\left(f-f_{h}\right) ; x\right)\right| & \leq\left\|f-f_{h}\right\| \\
& \leq \omega_{2}(f, h) .
\end{aligned}
$$

Now by Taylor's expansion and applying the Cauchy-Schwarz inequality, we have

$$
\begin{aligned}
\left|\mathcal{L}_{n}^{\rho}\left(f_{h}(t)-f_{h}(x) ; x\right)\right| & \leq\left|\mathcal{L}_{n}^{\rho}\left((t-x) f_{h}^{\prime}(x) ; x\right)\right|+\left|\mathcal{L}_{n}^{\rho}\left(\int_{x}^{t}(t-u) f_{h}^{\prime \prime}(u) d u ; x\right)\right| \\
& \leq\left\|f_{h}^{\prime}\right\|\left|\mathcal{L}_{n}^{\rho}(|t-x| ; x)\right|+\left\|f_{h}^{\prime \prime}\right\| \mathcal{L}_{n}^{\rho}\left(\left|\int_{x}^{t}\right| t-u|d u| ; x\right) \\
& =\left\|f_{h}^{\prime}\right\| \sqrt{\mathcal{L}_{n}^{\rho}\left((t-x)^{2} ; x\right)}+\frac{1}{2}\left\|f_{h}^{\prime \prime}\right\| \mathcal{L}_{n}^{\rho}\left((t-x)^{2} ; x\right) .
\end{aligned}
$$

Applying Lemma 2.2, equations (13), (14) and choosing $h$ as $\delta_{h, \rho}(x)$, we get the required result.

Theorem 3.3 For $f \in C_{E}^{2}\left(R_{0}^{+}\right)$, we obtain

$$
\lim _{n \rightarrow \infty} n\left[\mathcal{L}_{n}^{\rho}(f ; x)-f(x)\right]=\frac{A_{t_{1}}(1,1)+A_{t_{2}}(1,1)}{A(1,1)} f^{\prime}(x)+\frac{x}{2}\left(1+\frac{1}{\rho}\right) f^{\prime \prime}(x),
$$

uniformly in $x \in[0, a], a>0$.

Proof By Taylor's expansion of $f$ for some fixed $x \in[0, a]$, we obtain

$$
f(t)-f(x)=(t-x) f^{\prime}(x)+\frac{1}{2}(t-x)^{2} f^{\prime \prime}(x)+\xi(t, x)(t-x)^{2},
$$

where $\xi(t, x) \in C_{E}\left(R_{0}^{+}\right)$and $\lim _{t \rightarrow x} \xi(t, x)=0$.

Hence by linearity of the operators $\mathcal{L}_{n}^{\rho}$, from equation (17), we get

$$
\begin{aligned}
n\left[\mathcal{L}_{n}^{\rho}(f ; x)-f(x)\right]= & n \mathcal{L}_{n}^{\rho}(t-x ; x) f^{\prime}(x)+\frac{1}{2} n \mathcal{L}_{n}^{\rho}\left((t-x)^{2} ; x\right) f^{\prime \prime}(x) \\
& +n \mathcal{L}_{n}^{\rho}\left(\xi(t, x)(t-x)^{2} ; x\right)
\end{aligned}
$$

Applying the Cauchy-Schwarz inequality in the last term of equation (18), we have

$$
n \mathcal{L}_{n}^{\rho}\left(\xi(t, x)(t-x)^{2} ; x\right) \leq \sqrt{n^{2} \mathcal{L}_{n}^{\rho}\left((t-x)^{4} ; x\right) \mathcal{L}_{n}^{\rho}\left(\xi^{2}(t, x) ; x\right)} .
$$


From Remark 2.3, it follows that

$$
\lim _{n \rightarrow \infty} n^{2} \mathcal{L}_{n}^{\rho}\left((t-x)^{4} ; x\right)=3 x^{2}\left(1+\frac{2}{\rho}+\frac{1}{\rho^{2}}\right),
$$

uniformly in $x \in[0, a]$.

Further, let $\xi^{2}(t, x)=v(t, x), x \geq 0$, then $v(t, x) \in C_{E}\left(R_{0}^{+}\right)$and hence from Theorem 1.1, we get

$$
\lim _{n \rightarrow \infty} \mathcal{L}_{n}^{\rho}\left(\xi^{2}(t, x) ; x\right)=\lim _{n \rightarrow \infty} \mathcal{L}_{n}^{\rho}(v(t, x) ; x)=v(x, x)=0
$$

Hence from equation (19), we obtain

$$
\lim _{n \rightarrow \infty}\left(n \mathcal{L}_{n}^{\rho}\left(\xi(t, x)(t-x)^{2} ; x\right)\right)=0
$$

uniformly in $x \in[0, a]$. Now taking the limit $n \rightarrow \infty$ in (18) and using Remark 2.3, we get the desired result. This completes the proof.

\section{Weighted approximation}

Let $\theta(x) \geq 1$ be a weight function on $R_{0}^{+}$. We consider the weighted space defined on $R_{0}^{+}$:

$$
B_{\theta}\left(R_{0}^{+}\right):=\left\{f:|f(x)| \leq M_{f} \theta(x), \forall x \in R_{0}^{+} \text {and } M_{f}>0\right\}
$$

Further, let

$$
C_{\theta}\left(R_{0}^{+}\right):=\left\{f \in B_{\theta}\left(R_{0}^{+}\right): f \text { is a continuous function on } R_{0}^{+}\right\}
$$

and

$$
C_{\theta}^{*}\left(R_{0}^{+}\right):=\left\{f \in C_{\theta}\left(R_{0}^{+}\right): \lim _{x \rightarrow \infty} \frac{f(x)}{\theta(x)}=K_{f}<\infty\right\} .
$$

We define the norm in the space $B_{\theta}\left(R_{0}^{+}\right)$as

$$
\|f\|_{\theta}=\sup _{x \in R_{0}^{+}} \frac{|f(x)|}{\theta(x)} .
$$

The usual modulus of continuity of the function $f$ on $[0, p]$ is defined as

$$
\omega_{p}(f ; \delta)=\sup _{|t-x| \leq \delta} \sup _{t, x \in[0, p]}|f(t)-f(x)| .
$$

Let us denote $\|\cdot\|_{C[a, b]}$ as the supremum norm on $[a, b]$. Throughout the paper we have taken $\theta(x)=1+x^{2}$.

Theorem 4.1 For $x \in[0, c]$ and $f \in C_{\theta}\left(R_{0}^{+}\right)$, we have

$$
\left\|\mathcal{L}_{n}^{\rho}(f ; \cdot)-f\right\|_{C[0, c]} \leq 4 M_{f}\left(1+c^{2}\right) \eta_{n, \rho}^{2}+2 \omega_{c+1}\left(f ; \eta_{n, \rho}\right)
$$

where $\eta_{n, \rho}^{2}=\max _{x \in[0, c]}\left(\mathcal{L}_{n}^{\rho}\left(\left(t-x^{2}\right) ; x\right)\right)$. 
Proof Let $x \in[0, c]$ and $t>c+1$ then $t-x>1$. Then, for $f \in C_{\theta}\left(R_{0}^{+}\right)$, we have

$$
\begin{aligned}
|f(t)-f(x)| & \leq M_{f}\left(2+t^{2}+x^{2}\right) \\
& =M_{f}\left(2+2 x^{2}+(t-x)^{2}+2 x(t-x)\right) \\
& \leq M_{f}(t-x)^{2}\left(3+2 x^{2}+2 x\right) \leq 4 M_{f}\left(1+x^{2}\right)(t-x)^{2} .
\end{aligned}
$$

For $x \in[0, c]$ and $t \in[0, c+1]$, we have

$$
|f(t)-f(x)| \leq \omega_{c+1}(f ;|t-x|) \leq\left(1+\frac{|t-x|}{\delta}\right) \omega_{c+1}(f ; \delta) .
$$

From equations (24) and (25), for $x \in[0, c]$ and $t \geq 0$, we have

$$
|f(t)-f(x)| \leq 4 M_{f}\left(1+x^{2}\right)(t-x)^{2}+\left(1+\frac{|t-x|}{\delta}\right) \omega_{c+1}(f ; \delta) .
$$

Applying the Cauchy-Schwarz inequality and choosing $\delta=\sqrt{\eta_{n, \rho}}$, we get

$$
\begin{aligned}
\left|\mathcal{L}_{n}^{\rho}(f ; x)-f(x)\right| & \leq 4 M_{f}\left(1+x^{2}\right) \mathcal{L}_{n}^{\rho}\left(\left(t-x^{2}\right) ; x\right)+\left(1+\frac{1}{\delta} \mathcal{L}_{n}^{\rho}(|t-x| ; x)\right) \omega_{c+1}(f ; \delta) \\
& \leq 4 M_{f}\left(1+c^{2}\right) \eta_{n, \rho}^{2}(c)+2 \omega_{c+1}\left(f ; \eta_{n, \rho}(c)\right) .
\end{aligned}
$$

This completes the proof.

Theorem 4.2 For $f \in C_{\theta}\left(R_{0}^{+}\right)$, we have

$$
\lim _{n \rightarrow \infty} \sup _{x \in R_{0}^{+}} \frac{\left|\mathcal{L}_{n}^{\rho}(f ; x)-f(x)\right|}{\left(1+x^{2}\right)^{1+\eta}}=0
$$

where $\eta$ is some positive constant.

Proof Since $|f(x)| \leq\|f\|_{\theta}\left(1+x^{2}\right)$, for fixed $y>0$, we may write

$$
\begin{aligned}
\sup _{x \in R_{0}^{+}} \frac{\left|\mathcal{L}_{n}^{\rho}(f ; x)-f(x)\right|}{\left(1+x^{2}\right)^{1+\eta}} \leq \sup _{x \in[0, y]} \frac{\left|\mathcal{L}_{n}^{\rho}(f ; x)-f(x)\right|}{\left(1+x^{2}\right)^{1+\eta}}+\sup _{x \in(y, \infty)} \frac{\left|\mathcal{L}_{n}^{\rho}(f ; x)-f(x)\right|}{\left(1+x^{2}\right)^{1+\eta}} \\
\leq\left\|\mathcal{L}_{n}^{\rho}(f ; \cdot)-f\right\|_{C[0, y]}+\frac{\|f\|_{\theta}}{\left(1+y^{2}\right)^{\eta}} \\
\\
+\|f\|_{\theta} \sup _{x \in(y, \infty)} \frac{\left|\mathcal{L}_{n}^{\rho}\left(1+t^{2} ; x\right)\right|}{\left(1+x^{2}\right)^{1+\eta}}
\end{aligned}
$$

Using Theorem 3.1, for a given $\epsilon>0$, there exists $k \in \mathbb{N}$ such that

$$
\left|\mathcal{L}_{n}^{\rho}\left(1+t^{2} ; x\right)-1+x^{2}\right|<\frac{\epsilon}{3\|f\|_{\theta}}, \quad \forall n \geq k .
$$

or

$$
\mathcal{L}_{n}^{\rho}\left(1+t^{2} ; x\right)<1+x^{2}+\frac{\epsilon}{3\|f\|_{\theta}}, \quad \forall n \geq k .
$$


Hence,

$$
\begin{aligned}
\|f\|_{\theta} \frac{\mathcal{L}_{n}^{\rho}\left(1+t^{2} ; x\right)}{\left(1+x^{2}\right)^{1+\eta}} & <\frac{\|f\|_{\theta}}{\left(1+x^{2}\right)^{1+\eta}}\left(1+x^{2}+\frac{\epsilon}{3\|f\|_{\theta}}\right) \\
& <\frac{\|f\|_{\theta}}{\left(1+y^{2}\right)^{\eta}}+\frac{\epsilon}{3}, \quad \forall n \geq k .
\end{aligned}
$$

Therefore,

$$
\|f\|_{\theta} \sup _{x \in[y, \infty)} \frac{\mathcal{L}_{n}^{\rho}\left(1+t^{2} ; x\right)}{\left(1+x^{2}\right)^{1+\eta}} \leq \frac{\|f\|_{\theta}}{\left(1+y^{2}\right)^{\eta}}+\frac{\epsilon}{3}, \quad \text { for all } n \geq k .
$$

Let us choose y so large that

$$
\frac{\|f\|_{\theta}}{\left(1+y^{2}\right)^{\eta}} \leq \frac{\epsilon}{6}
$$

Also, in view of Theorem 4.1, for $\epsilon>0$ there exists a $n \geq l$ such that

$$
\left\|\mathcal{L}_{n}^{\rho}(f ; \cdot)-f\right\|_{C[0, y]}<\frac{\epsilon}{3}, \quad n \geq l .
$$

Taking $m=\max (k, l)$ and combining equations (26)-(29), we get

$$
\sup _{x \in R_{0}^{+}} \frac{\left|\mathcal{L}_{n}^{\rho}(f ; x)-f(x)\right|}{\left(1+x^{2}\right)^{1+\eta}}<\epsilon, \quad n \geq m .
$$

This completes the proof.

Following [13], the weighted modulus of continuity $\bar{\omega}(g ; \delta)$ for $g \in C_{\theta}\left(R_{0}^{+}\right)$is defined as

$$
\bar{\omega}(g ; \delta)=\sup _{0<|h| \leq \delta, x \in R_{0}^{+}} \frac{|g(x+h)-g(x)|}{\left(1+h^{2}\right)\left(1+x^{2}\right)} .
$$

Also, for $g \in C_{\theta}^{*}\left(R_{0}^{+}\right)$, the weighted modulus of continuity has the following properties:

$$
\lim _{\delta \rightarrow 0} \bar{\omega}(g ; \delta)=0
$$

and

$$
\bar{\omega}(g ; \lambda \delta) \leq 2(1+\lambda)\left(1+\delta^{2}\right) \bar{\omega}(g ; \delta), \quad \lambda>0
$$

For $g \in C_{\theta}\left(R_{0}^{+}\right)$, from equations (30) and (31)

$$
\begin{aligned}
|g(t)-g(x)| & \leq\left(1+(t-x)^{2}\right)\left(1+x^{2}\right) \bar{\omega}(g ;|t-x|) \\
& \leq 2\left(1+\frac{|t-x|}{\delta}\right)\left(1+\delta^{2}\right) \bar{\omega}(g ; \delta)\left(1+(t-x)^{2}\right)\left(1+x^{2}\right) .
\end{aligned}
$$


Theorem 4.3 For $f \in C_{\theta}^{*}\left(R_{0}^{+}\right)$, we have

$$
\sup _{x \in R_{0}^{+}} \frac{\left|\mathcal{L}_{n}^{\rho}(f ; x)-f(x)\right|}{\left(1+x^{2}\right)^{2}} \leq C \bar{\omega}\left(f ; \sqrt{\frac{1}{n}}\right),
$$

where $C$ is a positive constant independent of $n$.

Proof By the linearity and positivity of the operators $\mathcal{L}_{n}^{\rho}$, we get

$$
\left|\mathcal{L}_{n}^{\rho}(f ; x)-f(x)\right| \leq \mathcal{L}_{n}^{\rho}(|f(t)-f(x)| ; x)
$$

Using equation (32) and the Cauchy-Schwarz inequality, we get

$$
\begin{aligned}
\left|\mathcal{L}_{n}^{\rho}(f ; x)-f(x)\right| \leq & 2\left(1+\delta^{2}\right) \bar{\omega}(f ; \delta)\left(1+x^{2}\right) \mathcal{L}_{n}^{\rho}\left(\left(1+\frac{|t-x|}{\delta}\right)\left(1+(t-x)^{2}\right) ; x\right) \\
\leq & 2\left(1+\delta^{2}\right) \bar{\omega}(f ; \delta)\left(1+x^{2}\right)\left\{\mathcal{L}_{n}^{\rho}(1 ; x)+\mathcal{L}_{n}^{\rho}\left((t-x)^{2} ; x\right)\right. \\
& \left.+\frac{1}{\delta} \mathcal{L}_{n}^{\rho}(|t-x| ; x)+\frac{1}{\delta} \mathcal{L}_{n}^{\rho}\left(|t-x|(t-x)^{2} ; x\right)\right\} \\
\leq & 2\left(1+\delta^{2}\right) \bar{\omega}(f ; \delta)\left(1+x^{2}\right)\left\{\mathcal{L}_{n}^{\rho}(1 ; x)+\mathcal{L}_{n}^{\rho}\left((t-x)^{2} ; x\right)\right. \\
& \left.+\frac{1}{\delta} \sqrt{\mathcal{L}_{n}^{\rho}\left((t-x)^{2} ; x\right)}+\frac{1}{\delta} \sqrt{\mathcal{L}_{n}^{\rho}\left((t-x)^{2} ; x\right)} \sqrt{\mathcal{L}_{n}^{\rho}\left((t-x)^{4} ; x\right)}\right\} .
\end{aligned}
$$

Using Lemma 2.2, we obtain

$$
\mathcal{L}_{n}^{\rho}\left((t-x)^{2} ; x\right) \leq C_{1} \frac{1}{n}\left(1+x^{2}\right)
$$

and

$$
\mathcal{L}_{n}^{\rho}\left((t-x)^{4} ; x\right) \leq C_{2} \frac{1}{n^{2}}\left(1+x^{2}\right)
$$

for some positive constants $C_{1}$ and $C_{2}$ dependent on $\rho$ and $A\left(t_{1}, t_{2}\right)$. Now combining equations (33)-(35) and taking $\delta=\sqrt{\frac{1}{n}}$, we have

$$
\begin{aligned}
\left|\mathcal{L}_{n}^{\rho}(f ; x)-f(x)\right| \leq & 2\left(1+\frac{1}{n}\right) \bar{\omega}\left(f ; \sqrt{\frac{1}{n}}\right)\left(1+x^{2}\right)\left\{1+C_{1} \frac{1}{n}\left(1+x^{2}\right)\right. \\
& \left.+\sqrt{C_{1}} \sqrt{\left(1+x^{2}\right)}+\sqrt{C_{1}} \sqrt{\left(1+x^{2}\right)} \sqrt{C_{2}} \sqrt{\left(1+x^{2}\right)}\right\} .
\end{aligned}
$$

Hence, we get

$$
\sup _{x \in R_{0}^{+}} \frac{\left|\mathcal{L}_{n}^{\rho}(f ; x)-f(x)\right|}{\left(1+x^{2}\right)^{2}} \leq C \bar{\omega}\left(f ; \sqrt{\frac{1}{n}}\right)
$$

where $C=2\left(1+M C_{1}+\sqrt{C_{1}}+\sqrt{C_{1}} \sqrt{C_{2}}\right)$. This completes the proof. 


\section{Quantitative Voronovskaya theorems}

In the following result, we discuss a quantitative Voronovskaja type theorem by using the weighted modulus of smoothness $\bar{\omega}(f ; \delta)$. Recently, many researchers [14-18] have made remarkable contributions in this area.

Theorem 5.1 For $f, f^{\prime}, f^{\prime \prime}$ in $C_{\theta}^{*}\left(R_{0}^{+}\right)$and any $x \in R_{0}^{+}$, we have

$$
\begin{aligned}
& \mid n\left(\mathcal{L}_{n}^{\rho}(f ; x)-f(x)\right)-f^{\prime}(x)\left(\frac{A_{t_{1}}(1,1)+A_{t_{2}}(1,1)}{A(1,1)}\right)-\frac{f^{\prime \prime}(x)}{2 !}\left[\frac{x}{n}\left(1+\frac{1}{\rho}\right)\right. \\
& \quad+\frac{1}{n^{2} A(1,1)}\left\{\left(1+\frac{1}{\rho}\right)\left(A_{t_{1}}(1,1)+A_{t_{2}}(1,1)\right)\right. \\
& \left.\left.\quad+A_{t_{1} t_{1}}(1,1)+2 A_{t_{1} t_{2}}(1,1)+A_{t_{2} t_{2}}(1,1)\right\}\right] \mid \\
& =O(1) \bar{\omega}\left(f^{\prime \prime} ; \frac{1}{\sqrt{n}}\right), \quad \text { as } n \rightarrow \infty .
\end{aligned}
$$

Proof Let $x, t \in R_{0}^{+}$, then, by Taylor's expansion, we have

$$
f(t)=f(x)+f^{\prime}(x)(t-x)+\frac{f^{\prime \prime}(x)}{2 !}(t-x)^{2}+E(t, x)
$$

where $E(t, x)=\frac{f^{\prime \prime}(\varphi)-f^{\prime \prime}(x)}{2 !}(t-x)^{2}$ and $\varphi$ lies between $t$ and $x$.

Now, we get

$$
\left|\mathcal{L}_{n}^{\rho}(f ; x)-f(x)-f^{\prime}(x) \mathcal{L}_{n}^{\rho}((t-x) ; x)-\frac{f^{\prime \prime}(x)}{2 !} \mathcal{L}_{n}^{\rho}\left((t-x)^{2} ; x\right)\right| \leq \mathcal{L}_{n}^{\rho}(|E(t, x)| ; x) .
$$

Multiplying by $n$ on both sides of the above inequality and using Lemma 2.2, we obtain

$$
\begin{aligned}
& \mid n\left(\mathcal{L}_{n}^{\rho}(f ; x)-f(x)\right)-f^{\prime}(x)\left(\frac{A_{t_{1}}(1,1)+A_{t_{2}}(1,1)}{A(1,1)}\right)-\frac{f^{\prime \prime}(x)}{2 !}\left[\frac{x}{n}\left(1+\frac{1}{\rho}\right)\right. \\
& \quad+\frac{1}{n^{2} A(1,1)}\left\{\left(1+\frac{1}{\rho}\right)\left(A_{t_{1}}(1,1)+A_{t_{2}}(1,1)\right)\right. \\
& \left.\left.\quad+A_{t_{1} t_{1}}(1,1)+2 A_{t_{1} t_{2}}(1,1)+A_{t_{2} t_{2}}(1,1)\right\}\right] \mid \\
& \leq n \mathcal{L}_{n}^{\rho}(|E(t, x)| ; x) .
\end{aligned}
$$

Using the property of weighted modulus of smoothness given by (32), we get

$$
\begin{aligned}
\left|\frac{f^{\prime \prime}(\varphi)-f^{\prime \prime}(x)}{2 !}\right| \leq & \frac{1}{2} \bar{\omega}\left(f^{\prime \prime} ;|\varphi-x|\right)\left(1+(\varphi-x)^{2}\right)\left(1+x^{2}\right) \\
\leq & \frac{1}{2} \bar{\omega}\left(f^{\prime \prime} ;|t-x|\right)\left(1+(t-x)^{2}\right)\left(1+x^{2}\right) \\
\leq & \left(1+\frac{(|t-x|)}{\delta}\right)\left(1+\delta^{2}\right) \bar{\omega}\left(f^{\prime \prime} ; \delta\right) \\
& \times\left(1+(t-x)^{2}\right)\left(1+x^{2}\right) .
\end{aligned}
$$


Also,

$$
\left|\frac{f^{\prime \prime}(\varphi)-f^{\prime \prime}(x)}{2 !}\right| \leq \begin{cases}2\left(1+\delta^{2}\right)^{2}\left(1+x^{2}\right) \bar{\omega}\left(f^{\prime \prime} ; \delta\right), & |t-x| \leq \delta \\ 2\left(1+\delta^{2}\right)^{2}\left(1+x^{2}\right) \frac{(t-x)^{2}}{\delta^{4}} \bar{\omega}\left(f^{\prime \prime} ; \delta\right), & |t-x| \geq \delta\end{cases}
$$

Now for $0<\delta<1$, we obtain

$$
\left|\frac{f^{\prime \prime}(\varphi)-f^{\prime \prime}(x)}{2 !}\right| \leq 8\left(1+x^{2}\right) \bar{\omega}\left(f^{\prime \prime} ; \delta\right)\left(1+\frac{(t-x)^{4}}{\delta^{4}}\right) .
$$

Therefore, we get

$$
|E(t, x)| \leq 8\left(1+x^{2}\right) \bar{\omega}\left(f^{\prime \prime} ; \delta\right)\left((t-x)^{2}+\frac{(t-x)^{6}}{\delta^{4}}\right) .
$$

Now by the linearity and positivity of the operator $\mathcal{L}_{n}^{\rho}$ and using Remark 2.3, for any $x \in R_{0}^{+}$, we obtain

$$
\begin{aligned}
\mathcal{L}_{n}^{\rho}(|E(t, x)| ; x) & \leq 8\left(1+x^{2}\right) \bar{\omega}\left(f^{\prime \prime} ; \delta\right)\left\{\mathcal{L}_{n}^{\rho}\left((t-x)^{2} ; x\right)+\frac{1}{\delta^{4}} \mathcal{L}_{n}^{\rho}\left((t-x)^{6} ; x\right)\right\} \\
& \leq 8\left(1+x^{2}\right) \bar{\omega}\left(f^{\prime \prime} ; \delta\right)\left\{O\left(\frac{1}{n}\right)+O\left(\frac{1}{n^{3}}\right)\right\}
\end{aligned}
$$

Choosing $\delta=\frac{1}{\sqrt{n}}$, we obtain

$$
\mathcal{L}_{n}^{\rho}(|E(t, x)| ; x) \leq 8\left(1+x^{2}\right) \bar{\omega}\left(f^{\prime \prime} ; n^{-\frac{1}{2}}\right) O\left(\frac{1}{n}\right)
$$

Hence combining (36) and (37), we reach the required result.

\section{Grüss-Voronovskaya-type theorem}

For the first time Gal and Gonska [19], studied the Grüss Voronovskaya type theorem for the Bernstein, Păltănea and Bernstein-Faber operators by means of the Grüss inequality which concerns the non-multiplicavity of these operators. For more papers in this direction we refer the reader to ( $c f$. [20-22] etc.) Next, we study the non-multiplicativity of the positive linear operator $\mathcal{L}_{n}^{\rho}$.

Theorem 6.1 For $f^{\prime}(x), g^{\prime}(x), f^{\prime \prime}(x), g^{\prime \prime}(x),(f g)^{\prime}(x),(f g)^{\prime \prime}(x) \in C_{\theta}^{*}\left(R_{0}^{+}\right)$, we have the following equality:

$$
\lim _{n \rightarrow \infty} n\left\{\mathcal{L}_{n}^{\rho}(f g ; x)-\mathcal{L}_{n}^{\rho}(f ; x) \mathcal{L}_{n}^{\rho}(g ; x)\right\}=x\left(1+\frac{1}{\rho}\right) f^{\prime}(x) g^{\prime}(x)
$$

Proof We have

$$
(f g)^{\prime \prime}(x)=f^{\prime \prime}(x) g(x)+2 f^{\prime}(x) g^{\prime}(x)+g^{\prime \prime}(x) f(x) .
$$


By making an appropriate arrangement, we get

$$
\begin{aligned}
& n\left\{\mathcal{L}_{n}^{\rho}((f g) ; x)-\mathcal{L}_{n}^{\rho}(f ; x) \mathcal{L}_{n}^{\rho}(g ; x)\right\} \\
&=n\left\{\mathcal{L}_{n}^{\rho}((f g) ; x)-f(x) g(x)-(f g)^{\prime}(x) \mathcal{L}_{n}^{\rho}(t-x ; x)-\frac{(f g)^{\prime \prime}(x)}{2 !} \mathcal{L}_{n}^{\rho}\left((t-x)^{2} ; x\right)\right. \\
&-g(x)\left(\mathcal{L}_{n}^{\rho}(f ; x)-f(x)-f^{\prime}(x)\right) \mathcal{L}_{n}^{\rho}(t-x ; x)-\frac{f^{\prime \prime}(x)}{2 !} \mathcal{L}_{n}^{\rho}\left((t-x)^{2} ; x\right) \\
&-\mathcal{L}_{n}^{\rho}(f ; x)\left(\mathcal{L}_{n}^{\rho}(g ; x)-g(x)-(g)^{\prime}(x)\right) \mathcal{L}_{n}^{\rho}(t-x ; x)-\frac{(g)^{\prime \prime}(x)}{2 !} \mathcal{L}_{n}^{\rho}\left((t-x)^{2} ; x\right) \\
&\left.\left.\left.+2 \frac{\mathcal{L}_{n}^{\rho}\left((t-x)^{2} ; x\right)}{2 !} f^{\prime}(x)\right)(g)^{\prime}(x)\right)+(g)^{\prime \prime}(x)\right) \frac{\mathcal{L}_{n}^{\rho}\left((t-x)^{2} ; x\right)}{2 !}\left(f(x)-\mathcal{L}_{n}^{\rho}(f ; x)\right) \\
&\left.\left.+(g)^{\prime}(x)\right) \mathcal{L}_{n}^{\rho}(t-x ; x)\left(f(x)-\mathcal{L}_{n}^{\rho}(f ; x)\right)\right\} .
\end{aligned}
$$

Applying Theorem 3.1, for each $x \in R_{0}^{+}, L_{n}^{\rho}(f ; x) \rightarrow f(x)$ as $n \rightarrow \infty$ and for $f^{\prime \prime} \in C_{\theta}^{*}\left(R_{0}^{+}\right)$, $x \in R_{0}^{+}$, by Theorem 5.1 , we have

$$
\lim _{n \rightarrow \infty}\left(\mathcal{L}_{n}^{\rho}(f ; x)-f(x)-f^{\prime}(x) \mathcal{L}_{n}^{\rho}((t-x) ; x)-\frac{f^{\prime \prime}(x)}{2 !} \mathcal{L}_{n}^{\rho}\left((t-x)^{2} ; x\right)\right)=0
$$

Therefore, using Remark 2.3, we get the desired result.

\section{Rate of approximation of functions having derivative of bounded variation}

In the last decade, the degree of approximation for the functions having a derivative of bounded variation has been studied by several researchers. Ispir et al. [23] considered the Kantorovich modification of Lupas operators based on Polya distributions and studied the rate of approximation of the functions having a derivative of bounded variation. For other significant contributions in this direction $c f$. [16, 24-27] etc. Motivated by these studies, we shall discuss the rate of approximation of functions with a derivative of bounded variation on $R_{0}^{+}$for the operators $\mathcal{L}_{n}^{\rho}$.

Let $\operatorname{DBV}\left(R_{0}^{+}\right)$be the subspace of $B_{\theta}\left(R_{0}^{+}\right)$of all absolutely continuous functions $f$ having a derivative $f^{\prime}$ equivalent with a function of bounded variation on every finite subinterval of $R_{0}^{+}$. We observe that the functions $f \in \mathrm{DBV}\left(R_{0}^{+}\right)$possess a representation

$$
f(x)=\int_{0}^{x} g(t) d t+f(0)
$$

where $g \in B V\left(R_{0}^{+}\right)$, i.e., $g$ is a function of bounded variation on every finite subinterval of $R_{0}^{+}$.

In order to discuss the approximation of functions with derivatives of bounded variation, we express the operators $\mathcal{L}_{n}^{\rho}$ in an integral form as follows:

$$
\mathcal{L}_{n}^{\rho}(f ; x)=\int_{0}^{\infty} K_{n}^{\rho}(x, t) f(t) d t
$$


where the kernel $K_{n}^{\rho}(x, t)$ is given by

$$
\begin{aligned}
K_{n}^{\rho}(x, t)= & \frac{e^{-n x}}{A(1,1)} \sum_{k_{1}} \sum_{k_{1}+k_{2} \geq 1} \frac{p_{k_{1}, k_{2}}\left(\frac{n x}{2}\right)}{k_{1} ! k_{2} !} \frac{n \rho e^{-n \rho t}(n \rho t)^{\left(k_{1}+k_{2}\right) \rho-1}}{\Gamma\left(k_{1}+k_{2}\right) \rho} \\
& +\frac{e^{-n x}}{A(1,1)} p_{0,0}\left(\frac{n x}{2}\right) \delta(t)
\end{aligned}
$$

$\delta(t)$ being the Dirac-delta function.

Lemma 7.1 For a fixed $x \in R_{0}^{+}$and sufficiently large $n$, we have

(i) $\xi_{n}^{\rho}(x, y)=\int_{0}^{y} K_{n}^{\rho}(x, t) d t \leq \frac{C\left(1+\frac{1}{\rho}\right)(1+x)}{n} \frac{1}{(x-y)^{2}}, \quad 0 \leq y<x$,

(ii) $1-\xi_{n}^{\rho}(x, z)=\int_{z}^{\infty} K_{n}^{\rho}(x, t) d t \leq \frac{C\left(1+\frac{1}{\rho}\right)(1+x)}{n} \frac{1}{(z-x)^{2}}, \quad x<z<\infty$.

Proof (i) Using Lemma 2.2, we get

$$
\begin{aligned}
\xi_{n}^{\rho}(x, y) & =\int_{0}^{y} K_{n}^{\rho}(x, t) d t \leq \int_{0}^{y}\left(\frac{x-t}{x-y}\right)^{2} K_{n}^{\rho}(x, t) d t \\
& \leq \mathcal{L}_{n}^{\rho}\left((t-x)^{2} ; x\right)(x-y)^{-2} \\
& \leq \frac{C\left(1+\frac{1}{\rho}\right)(1+x)}{n} \frac{1}{(x-y)^{2}} .
\end{aligned}
$$

The proof of (ii) is similar; hence the details are omitted.

Theorem 7.2 Let $f \in \operatorname{DBV}\left(R_{0}^{+}\right)$. Then, for every $x \in R_{0}^{+}$and sufficiently large $n$, we have

$$
\begin{aligned}
& \left|\mathcal{L}_{n}^{\rho}(f ; x)-f(x)\right| \\
& \leq \frac{1}{2}\left(f^{\prime}(x+)+f^{\prime}(x-)\right)\left(\frac{A_{t_{1}}(1,1)+A_{t_{2}}(1,1)}{n A(1,1)}\right)+\frac{1}{2}\left|f^{\prime}(x+)-f^{\prime}(x-)\right| \sqrt{\frac{C}{n}\left(1+\frac{1}{\rho}\right)(1+x)} \\
& \quad+\frac{C}{n}\left(1+\frac{1}{\rho}\right) \frac{(1+x)}{x^{2}}\left|f(2 x)-f(x)-x f^{\prime}(x+)\right|+\frac{x}{\sqrt{n}} \bigvee_{x}^{x+x / \sqrt{n}}\left(f_{x}^{\prime}\right) \\
& \quad+\frac{C}{n}\left(1+\frac{1}{\rho}\right)\left(1+\frac{1}{x}\right) \sum_{k=1}^{[\sqrt{n}]} \bigvee_{x}^{x+x / \sqrt{k}} f_{x}^{\prime}+\frac{C}{n}\left(1+\frac{1}{\rho}\right)(1+x)\left(\frac{M+|f(x)|}{x^{2}}+4 M\right) \\
& \quad+\left|f^{\prime}(x+)\right| \sqrt{\frac{C}{n}\left(1+\frac{1}{\rho}\right)(1+x)},
\end{aligned}
$$

where $\bigvee_{a}^{b} f(x)$ denotes the total variation of $f(x)$ on $[a, b]$ and $f_{x}^{\prime}$ is defined by

$$
f_{x}^{\prime}(t)= \begin{cases}f^{\prime}(t)-f^{\prime}(x-), & 0 \leq t<x \\ 0, & t=x, \\ f^{\prime}(t)-f^{\prime}(x+) & x<t<\infty\end{cases}
$$


Proof Since $\mathcal{L}_{n}^{\rho}(1 ; x)=1$, using (38), for every $x \in(0,1)$ we get

$$
\begin{aligned}
\mathcal{L}_{n}^{\rho}(f ; x)-f(x) & =\int_{0}^{\infty} K_{n}^{\rho}(x, t)(f(t)-f(x)) d t \\
& =\int_{0}^{\infty} K_{n}^{\rho}(x, t) \int_{x}^{t} f^{\prime}(u) d u d t .
\end{aligned}
$$

For any $f \in \operatorname{DBV}\left(R_{0}^{+}\right)$, from (39) we may write

$$
\begin{aligned}
f^{\prime}(v)= & \left(f^{\prime}\right)_{x}(v)+\frac{1}{2}\left(f^{\prime}(x+)+f^{\prime}(x-)\right)+\frac{1}{2}\left(f^{\prime}(x+)-f^{\prime}(x-)\right) \operatorname{sgn}(v-x) \\
& +E_{x}(v)\left[f^{\prime}(v)-\frac{1}{2}\left(f^{\prime}(x+)+f^{\prime}(x-)\right)\right],
\end{aligned}
$$

where

$$
E_{x}(u)= \begin{cases}1, & v=x \\ 0, & v \neq x .\end{cases}
$$

We get

$$
\int_{0}^{\infty}\left(\int_{x}^{t}\left(f^{\prime}(u)-\frac{1}{2}\left(f^{\prime}(x+)+f^{\prime}(x-)\right)\right) E_{x}(v) d v\right) K_{n}^{\rho}(x, t) d t=0 .
$$

Using Lemma 2.2 and applying the Cauchy-Schwarz inequality, we obtain

$$
\begin{aligned}
& \int_{0}^{\infty}\left(\int_{x}^{t} \frac{1}{2}\left(f^{\prime}(x+)+f^{\prime}(x-)\right) d v\right) K_{n}^{\rho}(x, t) d t \\
& \quad=\frac{1}{2}\left(f^{\prime}(x+)+f^{\prime}(x-)\right) \int_{0}^{\infty}(t-x) K_{n}^{\rho}(x, t) d t \\
& \quad=\frac{1}{2}\left(f^{\prime}(x+)+f^{\prime}(x-)\right) \mathcal{L}_{n}^{\rho}((t-x) ; x)=\frac{1}{2}\left(f^{\prime}(x+)+f^{\prime}(x-)\right)\left(\frac{A_{t_{1}}(1,1)+A_{t_{2}}(1,1)}{n A(1,1)}\right)
\end{aligned}
$$

and

$$
\begin{aligned}
& \int_{0}^{\infty} K_{n}^{\rho}(x, t)\left(\int_{x}^{t} \frac{1}{2}\left(f^{\prime}(x+)-f^{\prime}(x-)\right) \operatorname{sgn}(v-x) d v\right) d t \\
& \quad \leq \frac{1}{2}\left|f^{\prime}(x+)-f^{\prime}(x-)\right|\left(\mathcal{L}_{n}^{\rho}\left((t-x)^{2} ; x\right)\right)^{1 / 2} \\
& \quad \leq \frac{1}{2}\left|f^{\prime}(x+)-f^{\prime}(x-)\right| \sqrt{\frac{C}{n}\left(1+\frac{1}{\rho}\right)(1+x) .}
\end{aligned}
$$

Using Lemma 2.2 and equations (40)-(44), we obtain

$$
\begin{aligned}
\left|\mathcal{L}_{n}^{\rho}(f ; x)-f(x)\right| \leq & \frac{1}{2}\left(f^{\prime}(x+)+f^{\prime}(x-)\right)\left(\frac{A_{t_{1}}(1,1)+A_{t_{2}}(1,1)}{n A(1,1)}\right) \\
& +\frac{1}{2}\left|f^{\prime}(x+)-f^{\prime}(x-)\right| \sqrt{\frac{C}{n}\left(1+\frac{1}{\rho}\right)(1+x)}+\left|I_{1}\right|+\left|I_{2}\right|,
\end{aligned}
$$


Ser and Agrawal Journal of Inequalities and Applications <wide>(2017<wide>) 2017:244

Page 17 of 20

where

$$
I_{1}=\int_{0}^{x} \int_{x}^{t}\left(\left(f^{\prime}\right)_{x}(v) d v\right) K_{n}^{\rho}(x, t) d t
$$

and

$$
I_{2}=\int_{x}^{1} \int_{x}^{t}\left(\left(f^{\prime}\right)_{x}(v) d v\right) K_{n}^{\rho}(x, t) d t
$$

Since we know $\int_{a}^{b} d_{t} \xi_{n}^{\rho}(x, t) \leq 1$, for all $[a, b] \subseteq R_{0}^{+}$, using integration by parts and applying Lemma 7.1 and substituting $y=x-x / \sqrt{n}$, we get

$$
\begin{aligned}
I_{1} & =\left|\int_{0}^{x} \int_{x}^{t}\left(\left(f^{\prime}\right)_{x}(v) d v\right) d_{t} \xi_{n}^{\rho}(x, t)\right| \\
& =\left|\int_{0}^{x} \xi_{n}^{\rho}(x, t)\left(f^{\prime}\right)_{x}(t) d t\right| \\
& \leq \int_{0}^{y}\left|\left(f^{\prime}\right)_{x}(t)\right|\left|\xi_{n}^{\rho}(x, t)\right| d t+\int_{y}^{x}\left|\left(f^{\prime}\right)_{x}(t)\right|\left|\xi_{n}^{\rho}(x, t)\right| d t \\
& \leq \frac{C}{n}\left(1+\frac{1}{\rho}\right)(1+x) \int_{0}^{y} \bigvee_{t}^{x}\left(\left(f^{\prime}\right)_{x}\right)(x-t)^{-2} d t+\int_{y}^{x} \bigvee_{t}^{x}\left(\left(f^{\prime}\right)_{x}\right) d t \\
& \leq \frac{C}{n}\left(1+\frac{1}{\rho}\right)(1+x) \int_{0}^{x-x / \sqrt{n}} \bigvee_{t}^{x}\left(\left(f^{\prime}\right)_{x}\right)(x-t)^{-2} d t+\frac{x}{\sqrt{n}} \bigvee_{x-x / \sqrt{n}}^{x}\left(\left(f^{\prime}\right)_{x}\right) .
\end{aligned}
$$

Substituting $v=x /(x-t)$, we get

$$
\begin{aligned}
& \frac{C}{n}\left(1+\frac{1}{\rho}\right)(1+x) \int_{0}^{x-x / \sqrt{n}}(x-t)^{-2} \bigvee_{t}^{x}\left(\left(f^{\prime}\right)_{x}\right) d t \\
& =\frac{C}{n}\left(1+\frac{1}{\rho}\right)(1+x) x^{-1} \int_{1}^{\sqrt{n}} \bigvee_{x-x / u}^{x}\left(\left(f^{\prime}\right)_{x}\right) d v \\
& \leq \frac{C}{n}\left(1+\frac{1}{\rho}\right)(1+x) x^{-1} \sum_{k=1}^{[\sqrt{n}]} \int_{k}^{k+1} \bigvee_{x-x / k}^{x}\left(\left(f^{\prime}\right)_{x}\right) d v \\
& \leq \frac{C}{n}\left(1+\frac{1}{\rho}\right)\left(1+\frac{1}{x}\right) \sum_{k=1}^{[\sqrt{n}]} \bigvee_{x-x / k}^{x}\left(\left(f^{\prime}\right)_{x}\right) .
\end{aligned}
$$

Thus,

$$
\left|I_{1}\right| \leq \frac{C}{n}\left(1+\frac{1}{\rho}\right)\left(1+\frac{1}{x}\right) \sum_{k=1}^{[\sqrt{n}]} \bigvee_{x-x / k}^{x}\left(\left(f^{\prime}\right)_{x}\right)+\frac{x}{\sqrt{n}} \bigvee_{x-x / \sqrt{n}}^{x}\left(\left(f^{\prime}\right)_{x}\right)
$$


Again, using integration by parts, applying the Cauchy-Schwarz inequality, Lemma 7.1 and substituting $z=x+(1-x) / \sqrt{n}$, we get

$$
\begin{aligned}
\left|I_{2}\right|= & \left|\int_{x}^{\infty} \int_{x}^{t}\left(\left(f^{\prime}\right)_{x}(v) d v\right) K_{n}^{\rho}(x, t) d t\right| \\
= & \left|\int_{x}^{2 x} \int_{x}^{t}\left(\left(f^{\prime}\right)_{x}(v) d v\right) d_{t}\left(1-\xi_{n}^{\rho}(x, t)\right)+\int_{2 x}^{\infty} \int_{x}^{t}\left(\left(f^{\prime}\right)_{x}(v) d v\right) K_{n}^{\rho}(x, t) d t\right| \\
= & \left|\left[\int_{x}^{t}\left(\left(f^{\prime}\right)_{x}(v) d v\right)\left(1-\xi_{n}^{\rho}(x, t)\right)\right]_{x}^{2 x}\right|+\left|\int_{x}^{2 x}\left(f^{\prime}\right)_{x}(t)\left(1-\xi_{n}^{\rho}(x, t)\right) d t\right| \\
& +\left|\int_{2 x}^{\infty} \int_{x}^{t}\left(\left(f^{\prime}(v)-f^{\prime}(x+)\right) d v\right) K_{n}^{\rho}(x, t) d t\right| \\
\leq & \left|\int_{x}^{2 x}\left(\left(f^{\prime}\right)_{x}(v) d v\right)\left(1-\xi_{n}^{\rho}(x, 2 x)\right)\right|+\left|\int_{x}^{2 x}\left(f^{\prime}\right)_{x}(t)\left(1-\xi_{n}^{\rho}(x, t)\right) d t\right| \\
& +\left|\int_{2 x}^{\infty} f(t) K_{n}^{\rho}(x, t) d t\right|+|f(x)|\left|\int_{2 x}^{\infty} K_{n}^{\rho}(x, t) d t\right| \\
& +\left|f^{\prime}(x+)\right|\left|\int_{2 x}^{\infty}((t-x)) K_{n}^{\rho}(x, t) d t\right| \\
\leq & \frac{C}{n}\left(1+\frac{1}{\rho}\right) \frac{(1+x)}{x^{2}} \mid \int_{x}^{2 x}\left(\left(f^{\prime}(v)-f(x+)\right) d v|+| \int_{x}^{x+x / \sqrt{n}} f_{x}^{\prime}(t) d t \mid\right. \\
& +\frac{C}{n}\left(1+\frac{1}{\rho}\right)(1+x)\left|\int_{x+x / \sqrt{n}}^{2 x}(t-x)^{-2} f_{x}^{\prime}(t) d t\right|+\left|\int_{2 x}^{\infty} f(t) K_{n}^{\rho}(x, t) d t\right| \\
& +|f(x)|\left|\int_{2 x}^{\infty} K_{n}^{\rho}(x, t) d t\right|+\left|f^{\prime}(x+)\right|\left(\int_{2 x}^{\infty}(t-x)^{2} K_{n}^{\rho}(x, t) d t\right)^{1 / 2} .
\end{aligned}
$$

By substituting $t=x+\frac{x}{u}$ and proceeding in a similar way to $I_{1}$, we get

$$
\begin{aligned}
\left|I_{2}\right| \leq & \frac{C}{n}\left(1+\frac{1}{\rho}\right) \frac{(1+x)}{x^{2}}\left|f(2 x)-f(x)-x f^{\prime}(x+)\right|+\frac{x}{\sqrt{n}} \bigvee_{x}^{x+x / \sqrt{n}}\left(f_{x}^{\prime}\right) \\
& +\frac{C}{n}\left(1+\frac{1}{\rho}\right)\left(1+\frac{1}{x}\right) \sum_{k=1}^{[\sqrt{n}]} \bigvee_{x}^{x+x / \sqrt{k}} f_{x}^{\prime}+\int_{2 x}^{\infty} M\left(1+t^{2}\right) K_{n}^{\rho}(x, t) d t \\
& +|f(x)|\left|\int_{2 x}^{\infty} K_{n}^{\rho}(x, t) d t\right|+\left|f^{\prime}(x+)\right| \sqrt{\frac{C}{n}\left(1+\frac{1}{\rho}\right)(1+x) .}
\end{aligned}
$$

Now for $t \geq 2 x$, we may write $t \leq 2(t-x)$ and $x \leq t-x$. Now using Lemma 7.1, we obtain

$$
\begin{aligned}
& \int_{2 x}^{\infty} M\left(1+t^{2}\right) K_{n}^{\rho}(x, t) d t+|f(x)| \int_{2 x}^{\infty} K_{n}^{\rho}(x, t) d t \\
& \quad \leq \frac{M}{x^{2}} \int_{2 x}^{\infty}(t-x)^{2} K_{n}^{\rho}(x, t) d t+4 M \int_{2 x}^{\infty}(t-x)^{2} K_{n}^{\rho}(x, t) d t
\end{aligned}
$$




$$
\begin{gathered}
+\frac{|f(x)|}{x^{2}} \int_{2 x}^{\infty}(t-x)^{2} K_{n}^{\rho}(x, t) d t \\
\leq \frac{C}{n}\left(1+\frac{1}{\rho}\right)(1+x)\left(\frac{M+|f(x)|}{x^{2}}+4 M\right) .
\end{gathered}
$$

Collecting the estimates (45)-(49), we get the required result.

\section{Conclusion}

A link between Szász-Durrmeyer type operators and multiple Appell polynomials has been established. The quantitative Voronovskaya type theorem and the GrüssVoronovskaya type theorem have been proved. A local approximation result and the weighted approximation theorem have been discussed besides the approximation of functions whose derivatives are locally of bounded variation.

\section{Acknowledgements}

The authors are extremely grateful to the reviewers for a critical reading of the manuscript and making valuable comments and suggestions leading to a better presentation of the paper. The first author is thankful to The Ministry of Human Resource and Development, India, for the financial support to carrying out the above work.

\section{Competing interests}

The authors declare that they have no competing interests.

\section{Authors' contributions}

All authors have equally contributed to this work. All authors read and approved the final manuscript.

\section{Publisher's Note}

Springer Nature remains neutral with regard to jurisdictional claims in published maps and institutional affiliations.

Received: 1 June 2017 Accepted: 18 September 2017 Published online: 03 October 2017

\section{References}

1. Szász, O: Generalization of S. Bernstein polynomials to the infinite interval. J. Res. Natl. Bur. Stand. 45, $239-245$ (1950)

2. Gupta, V, Kim, T, Lee, S-H: q-analogue of a new sequence of linear positive operators. J. Inequal. Appl. 2012, 144 (2012)

3. Gupta, V, Kim, T, Singh, VK, Lee, B: Rate of simultaneous approximation for Baskakov-Szász operators. Proc. Jangjeon Math. Soc. 14, 267-275 (2011)

4. Gupta, V, Sharma, H, Kim, T, Lee, S-H: Properties of q-analogue of Beta operator. Adv. Differ. Equ. 2012, $86(2012)$

5. Aral, A, Gupta, V, Agarwal, RP: Applications of q-Calculus in Operator Theory. Springer, New York (2013)

6. Gupta, V, Agrawal, RP: Convergence Estimates in Approximation Theory. Springer, New York (2014)

7. Gupta, V, Tachev, G: Approximation with Positive Linear Operators and Linear Combinations. Springer, Berlin (2017). ISBN 978-3-319-58794-3

8. Lee, DW: On multiple Appell polynomials. Proc. Am. Math. Soc. 139, 2133-2141 (2011)

9. Jakimovski, A, Leviatan, D: Generalized Szász operators for the approximation in the infinite interval. Mathematica 11, 97-103 (1969)

10. Varma, S: On a generalization of Szász operators by multiple Appell polynomials. Stud. Univ. Babeş-Bolyai, Math. 58, 361-369 (2013)

11. Páltánea, R: Modified-Szász-Mirakjan operators of integral form. Carpath. J. Math. 24, 378-385 (2008)

12. Gupta, V: (p,q)-Szász Mirakyan-Baskakov operators. Complex Anal. Oper. Theory (2015). doi:10.1007/s11785-015-0521-4

13. Ispir, N: On modified Baskakov operators on weighted spaces. Turk. J. Math. 25, 355-365 (2001)

14. Acar, T, Aral, A, Rasa, I: The new forms of Voronovoskaya's theorem in weiighted spaces. Positivity 20, 25-40 (2016)

15. Acar, T, Agrawal, PN, Neer, T: Bezier variant of the Bernstein-Durrmeyer type operators. Results Math. doi:10.1007/s00025-016-0639-3

16. Acar, T, Gupta, V, Aral, A: Rate of convergence for generalized Szasz operators. Bull. Math. Sci. 1, 99-113 (2011)

17. Acu, AM, Gonska, H, Rasa, I: Grüss-type and Ostrowski-type inequalities in approximation theory. Ukr. Math. J. 63, 843-864 (2011)

18. Acu, AM, Agrawal, PN, Neer, T: Approximation properties of modified Stancu operators. Numer. Funct. Anal. Optim. 38, 279-292 (2017)

19. Gal, SG, Gonska, H: Grüss and Grüss-Voronovskaya-type estimates for some Bernstein-type polynomials of real and complex variables. Jaen J. Approx. 7, 97-122 (2015)

20. Acar, T: Quantitative q-Voronovskaya and q-Grüss-Voronovskaya-type results for q-Szász operators. Georgian Math. J. 23, 459-468 (2016)

21. Deniz, E: Quantitative estimates for Jain-Kantorovich operators. Commun. Fac. Sci. Univ. Ank. Sér. A1 Math. Stat. 65 , $121-132(2016)$ 
22. Ulusoy, G, Acar, T: q-Voronovskaya type theorems for q-Baskakov operators. Math. Methods Appl. Sci. (2015). doi:10.1002/mma.3785

23. Ispir, N, Agrawal, PN, Kajla, A: Rate of convergence of Lupas Kantorovich operators based on Polya distribution. Appl. Math. Comput. 261, 323-329 (2015)

24. Agrawal, PN, Gupta, V, Kumar, AS, Kajla, A: Generalized-Baskakov Szász type operators. Appl. Math. Comput. 236, 311-324 (2014)

25. Cárdenas, D, Gupta, V: Two families of Bernstein-Durrmeyer type operators. Appl. Math. Comput. 248, 342-353 (2014)

26. Karsli, $\mathrm{H}$ : Rate of convergence of new gamma type operators for functions with derivatives of bounded variation. Math. Comput. Model. 45, 617-624 (2007)

27. Maheshwari, P: On modified Srivastava-Gupta operators. Filomat 29, 1173-1177 (2015)

\section{Submit your manuscript to a SpringerOpen ${ }^{\circ}$} journal and benefit from:

- Convenient online submission

- Rigorous peer review

- Open access: articles freely available online

- High visibility within the field

- Retaining the copyright to your article

Submit your next manuscript at $\gg$ springeropen.com 